\title{
Taux d'infestation et morphologie de la pyrale des dattes Ectomyelois ceratoniae (Zeller) sur différentes variétés du palmier dattier Phoenix dactylifera (L.)
}

\author{
Mohamed Azzedine Idder ${ }^{1}$ \\ Hakima Idder-Ighili ${ }^{1}$ \\ Hayet Saggou ${ }^{1}$ \\ Bernard Pintureau \\ ${ }^{1}$ Laboratoire de protection des écosystèmes \\ en zones arides et semi-arides \\ Université KASDI Merbah \\ BP 511 \\ 30000 Ouargla \\ Algérie \\ <azzou.idder@yahoo.fr> \\ 2 Inra \\ BF-2I \\ UMR Insa/Inra \\ Bâtiment Louis-Pasteur \\ Villeurbanne \\ F-69681 \\ France \\ <bernard.pintureau@lyon.inra.fr>
}

\begin{abstract}
Résumé
L'infestation des dattes par Ectomyelois ceratoniae dans les palmeraies de la région de Ouargla (sud-est algérien) varie selon la variété de la plante. Parmi les 13 variétés étudiées, la variété Takermoust est la plus infestée avec des taux pouvant atteindre $57 \%$, et les variétés Ben-Azizi, Ghars, et Tafezouine sont les moins infestées avec des taux ne dépassant pas 2 à $3 \%$. La pyrale a donc un impact plus ou moins grand selon les parcelles et/ou les variétés de palmiers dattiers. Ce résultat ouvre de nouvelles perspectives pour la lutte contre ce ravageur. Inversement, la variété de datte a une influence sur les pyrales. Ainsi, la taille du papillon est corrélée positivement à la taille du fruit, et probablement aussi soumise à d'autres facteurs comme la forme ou la qualité nutritive de ce fruit. La teinte du papillon, plus ou moins blanchâtre, varie également selon les variétés en fonction de la couleur de la datte.
\end{abstract}

Mots clés : Algérie ; infestation ; insecte nuisible ; morphologie ; palmier-dattier.

Thèmes : pathologie ; productions végétales.

\begin{abstract}
Infestation rate and morphology of the carob moth, Ectomyelois ceratoniae (Zeller), on different varieties of the palm date, Phoenix dactylifera (L.)

Ectomyelois ceratoniae is a more or less harmful pest for Wargla region dates (south eastern part of Algeria) according to the varieties of palm dates. Among the 13 studied varieties, the Takermoust variety is the most infected, the rate of damaged fruits reaching 57\%, and the Ben-Azizi, Ghars and Tafezouine varieties are the less infected, with rates of damaged fruits reaching only 2 to $3 \%$. Thus, the carob moth has a more or less important impact according to the cultivated plot and/or the palm date varieties. This result ushers in new possibilities of pest control. In parallel, date variety has an effect on E. ceratoniae. That is to say, the size of the moth is positively correlated to fruit size and probably also influenced by other factors such as the shape and nutritive quality of the fruit. The shade of the moth, more or less whitish, varies also according to the palm date variety in relation to the date colour.
\end{abstract}

Key words: Algeria; date palms; infestation; morphology; pest insects.

Subjects: pathology; vegetal productions. es travaux d'inventaire variétal, réalisés dans une quinzaine de régions algériennes, ont montré que les palmeraies présentent une importante diversité. En effet, 940 variétés ont été recensées (Hannachi et al., 1998), dont 270 dans la seule région Sud-Ouest (Ben Khalifa, 1989). La plus répandue est la variété Takermoust, seule résistante au Bayoud, pathologie induite par le cham- 
pignon Fusarium oxysporum. Au Sud-Est de l'Algérie, la diversité variétale est moins grande. Dans cette région prédomine la variété Deglet-Nour qui a une grande valeur marchande. On trouve aussi d'autres variétés plus ou moins abondantes telles que les variétés Ghars, Degla-Beida et Mech-Degla.

Les variétés sont essentiellement définies d'après les caractéristiques du fruit et seuls les individus femelles sont donc identifiables. Le terme " cultivar " est alors parfois préféré, surtout lorsqu'on parle de palmiers mâles (Bouguedoura, 1991).

Chaque variété de dattier présente le plus souvent une aire d'adaptation très marquée. C'est ainsi que la Mech-Degla de la région du Ziban n'est pas productive dans l'Oued Rhir, et inversement la Degla-Beida de l'Oued Rhir n'est pas productive au Ziban (Giovanni, 1969).

Cette richesse variétale est toutefois sujette à une érosion suite à différents facteurs : dégradation progressive de la palmeraie traditionnelle, vieillissement des palmeraies, déficit hydrique, maladie du Bayoud, exode rural et orientation vers la culture monovariétale (Belguedj, 2002). Les prospections faites dans la zone de Ouargla ont permis de recenser et d'échantillonner 58 variétés, mais plus de la moitié est menacée de disparition, car $90 \%$ des variétés rares sont composées d'individus âgés (Hannachi et Khitri, 1991).

La pyrale Ectomyelois ceratoniae est actuellement considérée comme le déprédateur le plus redoutable des dattes et comme la principale contrainte à l'exportation (Doumandji, 1981; DoumandjiMitiche, 1983; Idder, 1984; Raache, 1990 ; Haddad, 2000). Le pourcentage de fruits attaqués est souvent supérieur à $10 \%$ et peut atteindre $30 \%$ en Afrique du Nord (Wertheimer, 1958). Au moment de la récolte, ce pourcentage peut même parfois atteindre $80 \%$. (Munier, 1973). À Ouargla, il a été constaté que le pourcentage de fruits attaqués était de 42,5\% au sol et augmentait dans les lieux de stockage jusqu'à 64,7\% (Doumandji-Mitiche, 1983). En fait, les dégâts occasionnés par la pyrale des dattes dans cette région sont en moyenne de $22 \%$, bien que ce taux puisse varier d'une variété à une autre et d'une année à une autre (Idder, 1984). Le taux d'infestation de la variété DegletNour varie ainsi, selon les études, de 22,5 à 67,5\% (Benaddoun, 1987; Raache, 1990 ; Haddad, 2000).

$E$. ceratoniae est une espèce répandue dans tout le bassin méditerranéen et no- tamment au Maroc, en Algérie, Tunisie, Libye et Égypte. Sa présence a aussi été signalée en Espagne, en Italie, en Grèce et en France (Le Berre, 1978). Le papillon mesure de 6 à $14 \mathrm{~mm}$ de longueur et de 24 à 26 mm d'envergure (Le Berre, 1978). Il s'agit d'une espèce polyvoltine, et quatre générations peuvent se succéder au cours de l'année lorsque les conditions sont favorables. Ce nombre de générations varie en fait de 1 à 4 en fonction des conditions climatiques et de la plante hôte (Wertheimer, 1958; Doumandji, 1981). C'est aussi une espèce très polyphage, le nombre de plantes hôtes reconnues étant de 49 espèces dans le monde, dont 32 en Algérie (Doumandji, 1981). Les principales espèces présentes en Algérie sont le caroubier Ceratonia siliqua(L.), le néflier du Japon Eriobotrya japonica (Thunb.) Lindl, l'oranger Citrus sinensis (L.) Osbeck, le grenadier Punica granatum (L.) et le palmier dattier Phoenix dactylifera (L.) (Doumandji, 1981).

Cette étude a pour objectif de préciser l'influence de la pyrale sur la production de dattes à Ouargla et de mesurer sa variabilité en fonction des variétés végétales échantillonnées dans quatre parcelles. Elle vise aussi à rechercher d'éventuels effets des variétés de dattes sur les pyrales, et plus précisément sur la taille et la teinte des papillons.

\section{Matériel et méthode}

\section{Matériel}

Le matériel végétal est représenté par 13 variétés de palmiers dattiers dont les caractéristiques végétatives sont consignées dans le tableau 1. L'ensemble de ces variétés n'est toutefois pas présent sur les quatre sites choisis pour notre étude (tableau 2). Nous avons en effet retenu des palmeraies à diversité variétale plus ou moins grande et des biotopes contrastés (palmeraies nouvelles à plantation régulière et palmeraies anciennes à plantation irrégulière)

Le choix des pieds étudiés a été basé sur des critères de vigueur et de taille. Nous avons retenu des pieds vigoureux ayant une hauteur comprise entre 3 et $4 \mathrm{~m}$. Ces pieds ont été repérés à l'aide de plaques métalliques numérotées.

\section{Méthode}

Un échantillonnage des dattes a été effectué sur chacun des trois stades phénologiques du fruit, c'est-à-dire le grossissement, le début de maturité et la fin de maturité. Il a, pour cela, été tenu compte des périodes de maturation de chaque variété de dattes.

\section{Tableau 1. Caractéristiques de la partie végétative des variétés de palmiers dattiers étudiées (gamme de variation en $\mathrm{cm}$ ).}

Table 1. Characteristics of the vegetative part of the palm date varieties studied (range of variation in $\mathrm{cm}$ ).

\begin{tabular}{llll}
\hline Variétés & $\begin{array}{l}\text { Longueur } \\
\text { de la palme }\end{array}$ & $\begin{array}{l}\text { Largeur } \\
\text { de la palme }\end{array}$ & $\begin{array}{l}\text { Largeur } \\
\text { du spadice }\end{array}$ \\
\hline Tamsrit $(n=3)$ & $380-580$ & $73-110$ & 220 \\
Deglet-Nour $(n=4)$ & $370-480$ & $85-145$ & $140-260$ \\
Ghars $(n=4)$ & $370-510$ & $60-95$ & 180 \\
Degla-Beida $(n=4)$ & $300-380$ & $80-85$ & $130-140$ \\
Tafezouine $(n=2)$ & $350-490$ & $75-115$ & $103-188$ \\
Takermoust $(n=3)$ & $460-570$ & $82-109$ & $135-220$ \\
Ticherwit $(n=2)$ & 340 & 92 & 150 \\
Timjouhart $(n=1)$ & 520 & 90 & 230 \\
Hamraya $(n=4)$ & 380 & 60 & 120 \\
Harchaya $(n=1)$ & 430 & 97 & 150 \\
Mizit $(n=2)$ & 220 & 75 & 170 \\
Bayd-Hmam $(n=1)$ & 380 & 64 & 160 \\
Ben Azizi $(n=1)$ & 360 & 65 & 125 \\
\hline
\end{tabular}

$n=$ nombre d'arbres sur lesquels les mesures ont été prises. Quatre palmes de la couronne extérieure (ayant donc atteint leur croissance maximale), correspondant aux quatre positions cardinales, ont été mesurées sur chaque arbre. Trois spadices ont été mesurés sur chaque régime, soit entre 30 et 36 spadices par arbre. 
Tableau 2. Présentation des parcelles expérimentales irriguées par submersion.

Table 2. Presentation of the experimental plots irrigated by submersion.

\begin{tabular}{|c|c|c|c|c|}
\hline Caractéristiques & Parcelle P1 & Parcelle P2 & Parcelle P3 & Parcelle P4 \\
\hline Localisation & $\begin{array}{l}\text { N'goussa ( } 24 \mathrm{~km} \\
\text { de la ville de Ouargla) }\end{array}$ & Ksar (centre de Ouargla) & $\begin{array}{l}\text { IAS }{ }^{a}(6 \mathrm{~km} \text { de la ville } \\
\text { de Ouargla) }\end{array}$ & $\begin{array}{l}\text { ITDAS }^{b}(27 \mathrm{~km} \\
\text { de la ville de Ouargla) }\end{array}$ \\
\hline Type de plantation & $\begin{array}{l}\text { Ancienne exploitation } \\
\text { privée }\end{array}$ & $\begin{array}{l}\text { Ancienne exploitation } \\
\text { privée }\end{array}$ & $\begin{array}{l}\text { Nouvelle exploitation } \\
\text { commerciale }\end{array}$ & $\begin{array}{l}\text { Nouvelle exploitation } \\
\text { commerciale }\end{array}$ \\
\hline Plantation & Irrégulière & Irrégulière & Régulière & Régulière \\
\hline Superficie exploitée (ha) & 1,5 & 0,5 & 7,2 & 2 \\
\hline Nombre total de pieds & 91 & 31 & 855 & 130 \\
\hline \multirow[t]{11}{*}{ Nombre de pieds par variété } & 29 Ghars & 14 Ghars & 479 Deglet-Nour & 120 Deglet-Nour \\
\hline & 24 Deglet-Nour & 2 Deglet-Nour & 196 Ghars & 10 Ghars \\
\hline & 2 Takermoust & 2 Ticherwit & 7 Hamraya & \\
\hline & 3 Mizit & 1 Takermoust & 18 Degla-Beida & \\
\hline & 3 Tafezouine & 1 Harchaya & 1 Bayd-Hmam & \\
\hline & 2 Tamsrit & 5 Dgouls & 1 Tamsrit & \\
\hline & 2 Ben-Azizi & 5 Djebbars & 18 Dokkars & \\
\hline & 1 Timjouhart & 1 Dokkars & 135 Djebbars & \\
\hline & 5 Dgouls & & & \\
\hline & 15 Djebbars & & & \\
\hline & 5 Dokkars & & & \\
\hline Écartement entre pieds (m) & 5 à 6 & 2 à 3 & 9 & 12 \\
\hline Hauteur des pieds (m) & 3 à 3,5 & 4 à 5 & 3,5 à 4 & 3,5 à 4 \\
\hline \multirow{2}{*}{$\begin{array}{l}\text { Autres espèces de la strate } \\
\text { arboricole }\end{array}$} & Grenadier & Grenadier & Grenadier & \\
\hline & Figuier & Figuier & Vigne & \\
\hline Strate herbacée & $\begin{array}{l}\text { Luzerne, sorgho, chou } \\
\text { fourrager }\end{array}$ & $\begin{array}{l}\text { Persil, menthe, épinard, } \\
\text { luzerne }\end{array}$ & Luzerne, courgette & $\begin{array}{l}\text { Cultures maraîchères } \\
\text { sous-serre }\end{array}$ \\
\hline Brise vent & Palmes sèches & Palmes sèches & Casuarina & Casuarina \\
\hline Entretien de la palmeraie & Peu entretenue & Non entretenue & Non entretenue & Entretenue \\
\hline Drainage & Fonctionnelle & Non fonctionnelle & Non fonctionnelle & Fonctionnelle \\
\hline
\end{tabular}

a Institut d'agronomie saharienne.

${ }^{\mathrm{b}}$ Institut technologique de développement de I'agriculture saharienne.

Au cours de chaque échantillonnage, nous avons prélevé 30 dattes par arbre étudié à partir de quatre régimes d'orientations différentes (nord, sud, est et ouest par rapport au tronc du palmier dattier), sauf dans six cas où le nombre de dattes a été de 20. Le nombre d'arbres retenus dans chaque parcelle a été de 12 à 69, dont 1 à 24 par variété étudiée (1 pour la variété Timjouhart à 6 pour les variétés Deglet-Nour et Ghars dans la parcelle P1, 1 pour les variétés Takermoust et Harchaya à 6 pour la variété Ghars en P2, 1 pour les variétés Bayd-Hman et Tamsrit à 24 pour les variétés Deglet-Nour et Ghars en P3, 6 pour la variété Ghars à 10 pour la variété DegletNour en P4). Ces échantillons, placés dans un sac en papier Kraft correspondant à un seul arbre, ont été transportés le jour même de la récolte au laboratoire.

Les dattes prélevées ont alors été ouvertes à l'aide d'un scalpel pour vérifier la pré- sence de larves ou de nymphes de la pyrale. Même si l'on rencontre quelquefois deux ou trois larves de la pyrale dans un fruit, il ne sort qu'un papillon par datte à cause des phénomènes de cannibalisme. L'ensemble des dattes de même maturité, provenant d'un arbre, ont été stockées dans un bocal fermé à l'aide d'une toile à mailles fines de façon à permettre leur aération et à éviter la fuite des papillons et d'éventuels parasitoïdes. Ces derniers ont été récupérés dans des tubes à essai et observés sous loupe binoculaire afin de les déterminer. L'ensemble des bocaux a été placé à la température ambiante du laboratoire qui a varié entre 18 et $25^{\circ} \mathrm{C}$. Nous avons calculé le pourcentage d'infestation des fruits à chacun de leurs stades phénologiques (pourcentage de dattes renfermant au moins une larve de pyrale), sur chaque arbre étudié. Les mêmes pourcentages ont ensuite été calculés sur chaque variété de palmier dattier, dans chaque parcelle étudiée.

Certaines des dattes précédentes, infestées et arrivées en fin de maturité, ont individuellement été placées dans des pots afin d'étudier les relations entre la taille des fruits et des papillons qui en sont issus. Ces dattes, dont le nombre a varié entre 1 et 10 selon les variétés étudiées, ont été prélevées sur un à trois arbres selon ces variétés. Nous avons mesuré la longueur et la largeur de ces fruits (tableau 3), à l'aide de papier millimétré. La longueur des papillons issus des fruits infestés a ensuite été mesurée dans les mêmes conditions. Plusieurs corrélations entre la longueur des papillons et certains caractères des fruits ont enfin été calculées.

Nous avons comparé visuellement la couleur des dattes des diverses variétés ( $t a$ bleau 3) de façon à décrire cette variabi- 
Tableau 3. Caractéristiques des fruits des variétés de palmiers dattiers étudiées.

Table 3. Fruit characteristics of the palm date varieties studied.

\begin{tabular}{|c|c|c|c|c|c|c|}
\hline Variétés & Période de maturité & Forme et taille & Couleur & Consistance & Plasticité & Goût \\
\hline Tamsrit & $\begin{array}{l}\text { Août } \\
\text { Septembre }\end{array}$ & Droite grande & $\begin{array}{l}\text { Rouge (G) } \\
\text { Noire (M) }\end{array}$ & Molle à demi-molle & Tendre & Parfumé \\
\hline Deglet-Nour & $\begin{array}{l}\text { Octobre } \\
\text { Novembre }\end{array}$ & Ovoïde grande & $\begin{array}{l}\text { Rouge (G) } \\
\text { Variable (M) }\end{array}$ & Demi-molle & Tendre & Parfumé \\
\hline Ghars & Juillet & Droite grande & $\begin{array}{l}\text { Jaune (G) } \\
\text { Marron (M) }\end{array}$ & Molle à demi-molle & Élastique & Parfumé \\
\hline Degla-Beida & Octobre & Droite grande & Jaune (G et $M$ ) & Sèche & Dure & Acidulé \\
\hline Tafezouine & $\begin{array}{l}\text { Août } \\
\text { Septembre }\end{array}$ & Droite grande & $\begin{array}{l}\text { Jaune (G) } \\
\text { Ambrée (M) }\end{array}$ & Demi-molle & Tendre & Parfumé \\
\hline Takermoust & Septembre & Ronde moyenne & $\begin{array}{l}\text { Jaune }(G) \\
\text { Noire }(M)\end{array}$ & Demi-molle & Tendre & Parfumé \\
\hline Ticherwit & Septembre & Ovoïde moyenne & $\begin{array}{l}\text { Rouge (G) } \\
\text { Noire (M) }\end{array}$ & Demi-molle & Tendre & Parfumé \\
\hline Timjouhart & Août & Ovoïde grande & $\begin{array}{l}\text { Rouge (G) } \\
\text { Noire (M) }\end{array}$ & Demi-molle & Tendre & Parfumé \\
\hline Hamraya & $\begin{array}{l}\text { Août } \\
\text { Septembre }\end{array}$ & Droite grande & $\begin{array}{l}\text { Rouge (G) } \\
\text { Marron (M) }\end{array}$ & Molle à demi-sèche & Tendre & Acidulé \\
\hline Harchaya & Septembre & Ovoïde petite & $\begin{array}{l}\text { Jaune (G) } \\
\text { Marron (M) }\end{array}$ & Demi-sèche à sèche & Tendre & Acidulé \\
\hline Mizit & Septembre & Ovoïde moyenne & $\begin{array}{l}\text { Jaune (G) } \\
\text { Marron (M) }\end{array}$ & Molle & Tendre & Parfumé \\
\hline Bayd-Hmam & $\begin{array}{l}\text { Septembre } \\
\text { Octobre }\end{array}$ & Ovoïde petite & $\begin{array}{l}\text { Jaune (G) } \\
\text { Ambrée (M) }\end{array}$ & Molle à demi-molle & Tendre & Parfumé \\
\hline Ben-Azizi & Septembre & Ovoïde grande & $\begin{array}{l}\text { Jaune (G) } \\
\text { Ambrée (M) }\end{array}$ & Demi-molle & Tendre & Parfumé \\
\hline
\end{tabular}

$\mathrm{G}=$ stade de grossissement ; $\mathrm{M}=$ stade de maturité.

lité. Mais le but était surtout de la confronter à la variabilité de la teinte des pyrales adultes issues de chaque variété et de rechercher d'éventuelles relations.

\section{Statistiques}

Afin de comparer les quatre parcelles, plantées de façon régulière ou irrégulière, nous avons effectué une Anova à deux facteurs (parcelle, degré de maturité des fruits) sur les taux d'infestation des dattes de chacune des variétés Deglet-Nour et Ghars, seules présentes dans toutes ces parcelles. Les taux pris en compte, transformés en arcsin, se rapportent à un arbre (pourcentages de fruits infestés sur chaque arbre). L'effectif a été de 42 palmiers Deglet-Nour et 42 palmiers Ghars répartis comme suit : six en $\mathrm{P} 1$, deux en P2, 24 en P3 et dix en P4 pour la variété Deglet-Nour, et six en P1, six en P2, 24 en P3 et six en P4 pour la variété Ghars. Lorsqu'une Anova a indiqué une différence significative due à un facteur, des tests de comparaison multiple des taux pris deux à deux, associés à cette Anova et ne nécessitant pas de correction des données, ont été effectués : test PLSD (Procedure of least significant difference) de Fisher (logiciel StatView 5.0).

La suite de l'analyse, portant sur la comparaison des variétés sera conduite à l'aide d'autres Anovas à deux facteurs (variété, degré de maturité des fruits) sur l'ensemble des données ou celles se rapportant à certaines parcelles, selon les résultats des analyses précédentes. L'effectif total est de 120 arbres, avec un maximum de 42 pour les variétés Deglet-Nour et Ghars, et un minimum de 1 pour les variétés Bayd-Hmam, Harchaya et Timjouhart. Comme précédemment, ces Anovas ont été accompagnées de tests PLSD de Fisher lorsque des différences significatives le justifiaient.

Le calcul des corrélations entre les tailles du fruit et du papillon a concerné, d'une part, la longueur, la largeur et le produit de la longueur et de la largeur du fruit et, d'autre part, la longueur du papillon.

\section{Résultats}

\section{Influence de la pyrale sur le palmier dattier}

L'Anova à deux facteurs (parcelle et maturité), menée sur la variété Deglet-Nour, ne permet de détecter ni différence de taux d'infestation entre les parcelles, ni interaction entre les facteurs parcelle et stade de maturité, mais montre une différence significative d'infestation selon la maturité du fruit (tableau 4). Pour la variété Ghars, le degré de maturité conduit aux mêmes différences, mais les parcelles divergent également. L'interaction entre les facteurs est ici significative, ce qui suggère que le taux d'infestation de cette variété varie avec le stade de maturité de façon différente selon les parcelles (tableau 4). Dans ces deux analyses, les différences entre l'infestation des fruits plus ou moins matures ne concernent que le stade de fin de maturité, plus infesté, et les deux autres stades: selon le test PLSD de 


\section{Tableau 4. Résultats des Anova à deux facteurs (parcelles, degré de maturité des fruits) effectués sur le taux d'infestation des fruits de deux variétés de palmier dattier.}

Table 4. 2-factor (plot, maturity of fruit) Anova results for fruit infestation rates of two palm date varieties.

\begin{tabular}{lllll}
\hline & & Parcelle & Maturité & Interaction \\
\hline Variété Deglet-Nour & F & 1,308 & 6,591 & 1,418 \\
$n=126$ & p & 0,2752 & 0,0020 & 0,2139 \\
Variété Ghars & F & 5,027 & 9,690 & 3,086 \\
$n=127$ & p & 0,0026 & 0,0001 & 0,0077 \\
\hline
\end{tabular}

$n=$ nombre d'arbres étudiés.

Fisher, $p<0,0001$ entre les stades de grossissement et de fin de maturité pour les deux variétés, et entre les stades de début et de fin de maturité pour la variété Deglet-Nour ; $p=0,003$ entre les stades de début et de fin de maturité pour la variété Ghars $(p>0,05$ entre les stades de grossissement et de début de maturité pour les deux variétés).

En ce qui concerne les différences entre parcelles pour la variété Ghars, celles-ci sont tout à fait modestes puisqu'elles ne portent que sur deux parcelles (une comparaison sur les six possibles) : la $\mathrm{P} 3,-$ non entretenue et avec une densité de 118,7 arbres/ha -, est plus infestée que la P4, - entretenue et qui présente une densité plus faible (65 arbres/ha) - $(p=0,004$ selon le test PLSD de Fisher). Dans une optique de simplification, nous avons donc décidé de regrouper l'ensemble des parcelles pour la suite des analyses visant à comparer les variétés. Une seule Anova a en conséquence été réalisée dans ce but. Cette analyse montre une différence significative entre les variétés $(p<0,0001)$ et les degrés de maturité des fruits $(p<0,0001)$, l'interaction entre les facteurs étant également significative $(p=0,029)$. Pour ce qui est de l'influence de la maturité des fruits, le test PLSD de Fisher montre les mêmes différences que dans les Anovas préliminaires $(p<0,0001)$.

Pour ce qui est de l'influence des variétés, le test PLSD de Fisher suggère l'existence d'au moins deux groupes, un groupe de variétés présentant de fortes infestations et un autre présentant des infestations moyennes à faibles (tableau 5). Le groupe le plus infesté comprend les variétés Takermoust, Timjouhart, Bayd-Hmam et Mizit avec des taux d'infestation des fruits en fin de maturité qui atteignent respectivement 57, 30, 20 et $15 \%$ (tableau 5). Le second groupe comprend des variétés moyennement infestées, telles que Deglet-Nour et Degla-Beida présentant des taux d'infestation des fruits matures pouvant atteindre respectivement 13,2 et $9,7 \%$, et des variétés très peu infestées, comme Ghars, Tafezouine et Ben-Azizi avec des taux d'infestation n'atteignant respectivement que $3,3,3,3$ et $1,7 \%$ (tableau 6).

\section{Influence du palmier dattier sur la pyrale}

Relation entre la taille des pyrales adultes et la taille des dattes de différentes variétés

La taille du fruit est très variable selon les variétés, la longueur allant de 2,5 à 4,9 cm et la largeur de 0,9 à 2,0 cm. La longueur du papillon varie également beaucoup puisque, selon les variétés de dattes, elle va de 0,7 à $1,3 \mathrm{~cm}$. Les moyennes par variété figurent dans le tableau 7 .

La plus forte corrélation $(p<0,01$, figure $1 B$ ) est constatée entre la longueur du

\section{Tableau 5. Seuils de signification des différences entre taux d'infestation des 13 variétés de dattes étudiées, d'après le test PLSD de Fisher, après une Anova à deux facteurs (variétés, degrés de maturité des fruits).}

Table 5. Significance of difference thresholds between rates of infestation of the 13 date varieties studied according to the Fisher PLSD test and after 2-factor (variety, maturity of fruit) Anova.

\begin{tabular}{|c|c|c|c|c|c|c|c|c|c|c|c|c|c|}
\hline Tak & Tim & $\mathrm{BH}$ & Miz & DN & DB & Ham & Har & Tam & Tic & Taf & Gha & BA & Variétés \\
\hline- & $\begin{array}{l}>0,05 \\
-\end{array}$ & $\begin{array}{l}>0,05 \\
>0,05 \\
-\end{array}$ & $\begin{array}{l}>0,05 \\
>0,05 \\
>0,05\end{array}$ & $\begin{array}{l}<0,01 \\
<0,01 \\
>0,05 \\
>0,05 \\
-\end{array}$ & $\begin{array}{l}<0,01 \\
<0,01 \\
>0,05 \\
0,02 \\
>0,05 \\
-\end{array}$ & $\begin{array}{l}<0,01 \\
<0,01 \\
0,04 \\
0,01 \\
>0,05 \\
>0,05 \\
-\end{array}$ & $\begin{array}{l}>0,05 \\
0,04 \\
>0,05 \\
>0,05 \\
>0,05 \\
>0,05 \\
>0,05 \\
-\end{array}$ & $\begin{array}{l}0,02 \\
0,01 \\
>0,05 \\
>0,05 \\
>0,05 \\
>0,05 \\
>0,05 \\
>0,05 \\
-\end{array}$ & $\begin{array}{l}>0,05 \\
0,03 \\
>0,05 \\
>0,05 \\
>0,05 \\
>0,05 \\
>0,05 \\
>0,05 \\
>0,05 \\
-\end{array}$ & $\begin{array}{l}0,03 \\
0,02 \\
>0,05 \\
>0,05 \\
>0,05 \\
>0,05 \\
>0,05 \\
>0,05 \\
>0,05 \\
>0,05 \\
-\end{array}$ & $\begin{array}{l}<0,01 \\
<0,01 \\
0,01 \\
<0,01 \\
<0,01 \\
>0,05 \\
>0,05 \\
>0,05 \\
>0,05 \\
>0,05 \\
>0,05 \\
-\end{array}$ & $\begin{array}{l}0,01 \\
<0,01 \\
>0,05 \\
0,05 \\
>0,05 \\
>0,05 \\
>0,05 \\
>0,05 \\
>0,05 \\
>0,05 \\
>0,05 \\
>0,05 \\
-\end{array}$ & $\begin{array}{l}\text { Takermoust (Tak) } \\
\text { Timjouhart (Tim) } \\
\text { Bayd-Hmam (BH) } \\
\text { Mizit (Miz) } \\
\text { Deglet-Nour (DN) } \\
\text { Degla-Beida (DB) } \\
\text { Hamraya (Ham) } \\
\text { Harchaya (Har) } \\
\text { Tamsrit (Tam) } \\
\text { Ticherwit (Tic) } \\
\text { Tafezouine (Taf) } \\
\text { Ghars (Gha) } \\
\text { Ben-Azizi (BA) }\end{array}$ \\
\hline
\end{tabular}

Les variétés les plus infestées se trouvent à gauche ou en haut. 
Tableau 6. Pourcentage de dattes de chaque variété infestées par la pyrale, dans les quatre parcelles P1 à P4.

Table 6. Percentages of dates from each variety suffering moth infestation in the four plots P1 to P4.

\begin{tabular}{|c|c|c|c|c|c|c|c|c|c|c|c|c|}
\hline \multirow[t]{2}{*}{ Variétés $(n)^{\mathrm{a}}$} & \multicolumn{3}{|c|}{ P1 } & \multicolumn{3}{|c|}{ P2 } & \multicolumn{3}{|c|}{ P3 } & \multicolumn{3}{|c|}{ P4 } \\
\hline & GF & DM & FM & GF & DM & FM & GF & DM & FM & GF & DM & FM \\
\hline $\begin{array}{l}\text { Deglet-Nour } \\
(180,60,720,300)\end{array}$ & 0 & 0,5 & 3,9 & 0 & 0 & 5,0 & 0 & 1,1 & 13,2 & 0 & 0 & 1,0 \\
\hline $\begin{array}{l}\text { Ghars } \\
(180,180,720,180)\end{array}$ & 0 & 1,1 & 1,1 & 0 & 0 & 3,3 & 0 & 0 & 2,2 & 0 & 0 & 0 \\
\hline $\begin{array}{l}\text { Ben-Azizi } \\
(60)\end{array}$ & 0 & 1,7 & 1,7 & - & - & - & - & - & - & - & - & - \\
\hline $\begin{array}{l}\text { Mizit } \\
(60)\end{array}$ & 0 & 1,7 & 15,0 & - & - & - & - & - & - & - & - & - \\
\hline $\begin{array}{l}\text { Tamsrit } \\
(60,30)\end{array}$ & 0 & 3,3 & 5,3 & - & - & - & 0 & 0 & 3,3 & & - & - \\
\hline $\begin{array}{l}\text { Tafezouine } \\
\text { (60) }\end{array}$ & 0 & 3,3 & 3,3 & - & - & - & - & - & - & - & - & - \\
\hline $\begin{array}{l}\text { Takermoust } \\
(60,30)\end{array}$ & 2,0 & 1,7 & 11,7 & 10,0 & 0 & 56,7 & - & - & - & - & - & - \\
\hline $\begin{array}{l}\text { Timjouhart } \\
\text { (30) }\end{array}$ & 0 & 10,0 & 30,0 & - & - & - & - & - & - & - & - & - \\
\hline $\begin{array}{l}\text { Ticherwit } \\
\text { (60) }\end{array}$ & - & - & - & 2,0 & 0 & 5,0 & - & - & - & - & - & - \\
\hline $\begin{array}{l}\text { Hamraya } \\
(260)\end{array}$ & - & - & - & & & & 0 & 0 & 6,7 & - & - & - \\
\hline $\begin{array}{l}\text { Degla-Beida } \\
(300)\end{array}$ & - & - & - & - & - & - & 0 & 6,4 & 9,7 & - & - & - \\
\hline $\begin{array}{l}\text { Harchaya } \\
\text { (30) }\end{array}$ & - & - & - & 0 & 0,4 & 6,0 & & & & - & - & - \\
\hline $\begin{array}{l}\text { Bayd-Hmam } \\
\text { (30) }\end{array}$ & - & - & - & - & - & - & 0 & 3,3 & 20,0 & - & - & - \\
\hline
\end{tabular}

GF : grossissement du fruit ; DM : début de maturité du fruit ; FM : fin de maturité du fruit.

${ }^{a}$ Respectivement, dans I'ordre, pour P1, P2, P3 et P4, les effectifs de dattes observées ont été les mêmes aux trois stades phénologiques.

\section{Tableau 7. Taille moyenne en $\mathbf{m m}$ ( \pm erreur standard) des papillons $d^{\prime} E$. ceratoniae et des dattes d'où ils sont issus.}

Table 7. Average size in $\mathrm{mm}$ ( \pm standard error) of $E$. ceratoniae moths and of the dates from which they come.

\begin{tabular}{|c|c|c|c|}
\hline Variétés & Longueur du fruit & Largeur du fruit & Longueur du papillon \\
\hline Tamsrit & $40,9 \pm 0,2$ & $16,6 \pm 0,4$ & $12,0 \pm 0,2$ \\
\hline Deglet-Nour & $35,9 \pm 0,3$ & $15,6 \pm 0,3$ & $10,6 \pm 0,2$ \\
\hline Ghars & $40,8 \pm 0,2$ & $17,1 \pm 0,2$ & $12,0 \pm 0,2$ \\
\hline Degla-Beida & $41,0 \pm 0,7$ & $16,5 \pm 0,4$ & $12,0 \pm 0,4$ \\
\hline Tafezouine & $37,5 \pm 0,7$ & $14,5 \pm 0,7$ & $11,5 \pm 0,7$ \\
\hline Takermoust & $29,8 \pm 1,7$ & $17,0 \pm 0,9$ & $12,2 \pm 0,5$ \\
\hline Ticherwit & $30,6 \pm 0,2$ & $13,1 \pm 0,2$ & $9,3 \pm 0,2$ \\
\hline Timjouhart & $41,0 \pm 2,0$ & $16,6 \pm 0,7$ & $12,0 \pm 0,4$ \\
\hline Hamraya & $37,1 \pm 1,4$ & $14,6 \pm 0,6$ & $11,4 \pm 0,5$ \\
\hline Harchaya & $25,5 \pm 0,7$ & $10,2 \pm 0,4$ & $7,5 \pm 0,7$ \\
\hline Mizit & $32,5 \pm 0,4$ & $14,0 \pm 0,7$ & $9,5 \pm 0,3$ \\
\hline Bayd-Hmam & $24,8 \pm 0,4$ & $9,8 \pm 0,4$ & $7,2 \pm 0,2$ \\
\hline Ben-Azizi & $36,4 \pm 0,2$ & $16,2 \pm 0,2$ & $11,1 \pm 0,2$ \\
\hline
\end{tabular}

Selon les variétés, l'échantillon a été collecté sur un à trois arbres, à raison d'un à cinq fruits par arbre. papillon et la largeur du fruit. Une corrélation plus faible mais tout aussi significative existe entre la longueur du papillon et le produit longueur $\times$ largeur du fruit (figure 1C) ou la longueur du fruit (figure 1A). Notons que la relation de la taille des papillons avec le log de la taille des fruits n'est guère plus explicative que la relation linéaire : $r=0,710$ pour la longueur des fruits, $r=0,858$ pour la largeur des fruits et $r=0,825$ pour le produit de la longueur par la largeur. Nous nous en tiendrons donc à la relation linéaire pour observer que dans le cas de la longueur du fruit, sept points s'éloignent nettement de la droite de régression, quatre au-dessus de la droite correspondent à la variété Takermoust (sur les cinq fruits étudiés) et trois au-dessous de la droite correspondent à la variété Bayd-Hmam (trois fruits étudiés). Cet écart pourrait être rapporté à la forme originale des fruits, ronde, dans le premier cas et à la taille des fruits, très petite, dans le second cas. 


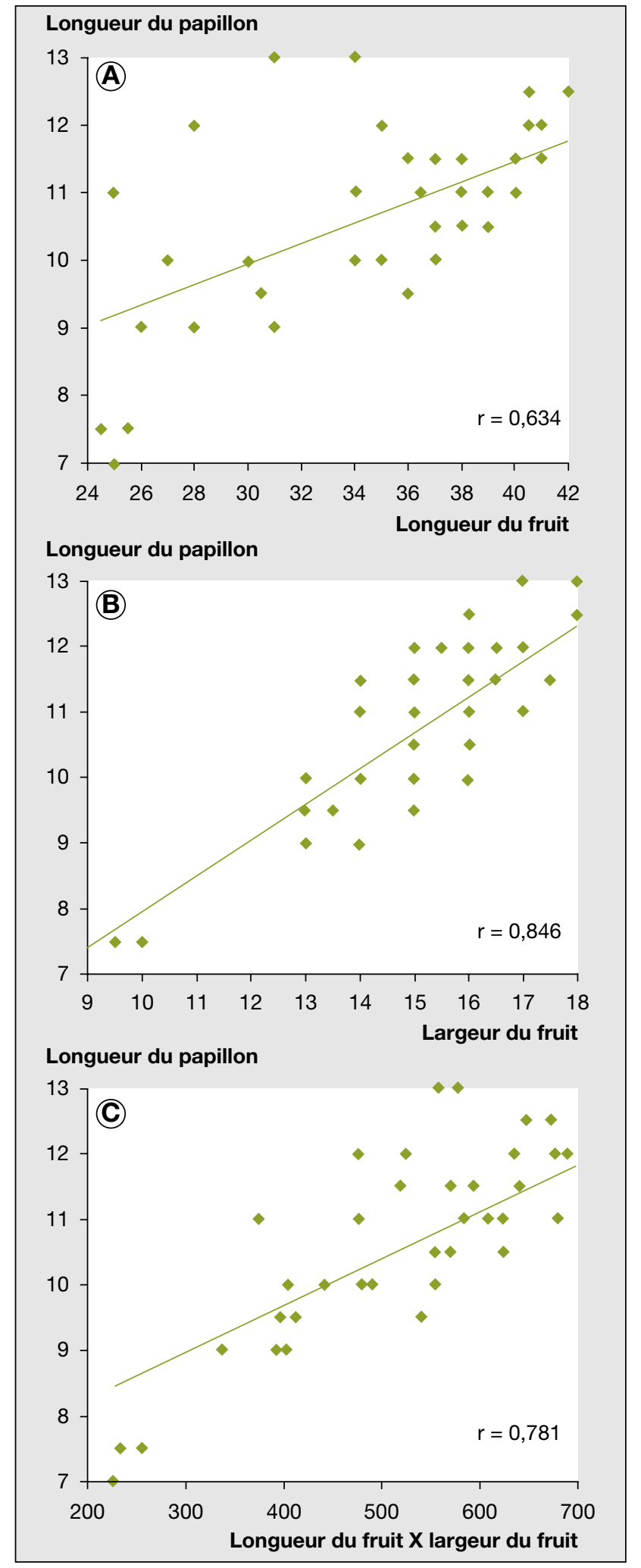

Figure 1. Régression entre la longueur des papillons $E$. ceratoniae et la longueur des fruits (A), la largeur des fruits $(B)$ ou le produit longueur des fruits $\times$ largeur des fruits d'où ils sont issus $(C)$.

Figure 1. Regression between the moth length (E. ceratoniae) and the size of the fruit from where the pest emerged: fruit length $(A)$, fruit width $(B)$ or "fruit length $\times$ fruit width".
Relation entre la teinte des pyrales et la teinte des dattes de différentes variétés

La coloration de la pyrale des dattes est très variable, ce qui a conduit certains auteurs à décrire des catégories systématiques distinctes (Pintureau et Daumal, 1979). En fait, cette espèce présente en Afrique du Nord un polymorphisme de coloration (déterminé génétiquement) et tous les intermédiaires entre un morphe foncé (ceratoniae) et un morphe clair (ponicis) existent, le premier étant mieux adapté aux biotopes comprenant des plantes hôtes telles que le caroubier, le figuier ou l'oranger, et le second aux biotopes comprenant des palmiers dattiers (Pintureau et Daumal, 1979 ; Doumandji, 1981). Rien n'indique toutefois l'existence de "races d'hôtes", puisque chaque morphe accepte toutes les nourritures utilisées par l'espèce (Pintureau et Daumal, 1979).

Mais des variations plus discrètes de teinte existent aussi chez un même morphe de pyrale en fonction de la variété de la datte infestée, et probablement aussi de l'espèce végétale hôte. Les nombreuses observations que nous avons effectuées (observation des mêmes effectifs de papillons et de dattes que dans l'étude précédente relative à la taille) montrent en effet une relation entre la teinte du ravageur et la coloration du fruit. Ainsi, les papillons issus de variétés telles que Takermoust, Tamsrit et Ticherwit, dont le fruit présente une coloration noire au stade de maturité, ont une teinte sombre. Les papillons issus de la variété Hamraya, dont le fruit est de couleur marron au stade de maturité, prennent une teinte moins foncée. Les papillons les plus clairs sont issus de la variété Degla-Beida au fruit jaune.

\section{Discussion}

\section{et conclusions}

Des auteurs ont suggéré que le taux d'infestation des dattes par E. ceratoniae est souvent plus élevé en palmeraies à plantations irrégulières qu'en palmeraies à plantations régulières (Benaddoun, 1987 ; Raache, 1990 ; Idder, 1992 ; Haddad, 2000). Dans le premier cas, la densité importante des arbres constituerait en effet un facteur favorable à la propagation du ravageur, la présence de plantes 
comme le figuier pouvant aussi contribuer à cette propagation. Parmi les quatre parcelles étudiées, dont l'infestation n'a pu être comparée qu'à partir de deux variétés, nous n'avons toutefois détecté de différence qu'entre deux parcelles à plantation régulière et cela pour une seule variété de dattes. C'est alors l'état d'entretien de la palmeraie qui a pu influer sur le taux d'infestation. La différence relevée concerne en effet la parcelle $\mathrm{P} 3$ non entretenue qui est plus infestée que la parcelle P4 entretenue. Ces parcelles se caractérisent toutefois par de nombreux autres facteurs, dont la diversité variétale et la densité des palmiers dattiers. L'absence d'entretien pourrait être favorable à la pyrale en offrant des refuges tels que des dattes tombées au sol, sur des cornafs (bases des palmes coupées) ou sur la couronne foliaire, et diverses plantes hôtes en plus des palmiers dattiers. La détermination des facteurs influant le plus sur l'infestation des palmeraies suppose toutefois d'entreprendre des échantillonnages dans un très grand nombre de parcelles se différenciant par le type de plantation, l'entretien de la plantation, la densité de plantation, la composition en plantes hôtes (espèces et variétés), voire le degré d'infestation des dattes par des champignons (Cossé et al., 1994).

Les dattes sont de plus en plus infestées en franchissant leurs trois stades phénologiques. Notre méthode d'observation semble exclure une moindre détection des chenilles dans les jeunes fruits et indique donc un réel phénomène biologique. Les papillons préfèreraient donc des dattes matures pour déposer leurs pontes, le fruit en fin de maturité constituant probablement un milieu nutritif mieux adapté aux exigences du déprédateur.

Si le taux d'infestation des dattes varie peu entre parcelles étudiées, il varie en revanche beaucoup entre variétés. L'hétérogénéité de la composition variétale dans les différentes parcelles a dû influer quelque peu sur l'infestation de chaque variété, mais elle n'a pas pu masquer le fait qu'il existe des variétés fortement attaquées et d'autres beaucoup mieux protégées. Ainsi, la variété Takermoust est parmi les plus infestées et la variété Ben-Azizi parmi les moins infestées. Seules trois variétés, Takermoust, Ticherwit et Tamsrit, ont montré des infestations précoces depuis le stade de grossissement des fruits. Le taux d'infestation plus élevé de certaines variétés de dattes pourrait être dû à une variabilité des substances volatiles émises, exerçant des effets plus ou moins accentués d'attractivité ou de répulsion. De telles substances pourraient non seulement provenir des fruits, mais aussi d'organismes associés (Cossé et al., 1994). Nous avons constaté qu'il existe une relation étroite entre la longueur des papillons et la taille des dattes, ce qui semble indiquer que plus la chenille dispose de nourriture, plus elle en consomme. Toutefois, la variété Takermoust fournit des papillons de taille relativement plus grande qu'attendue au regard de cette relation, et il se pourrait que la forme ronde du fruit en soit responsable. Au contraire, la variété Bayd-Hmam fournit des papillons de petite taille, et il se pourrait que la très petite taille du fruit en soit responsable. Ces deux seules exceptions que nous avons constatées à la relation entre tailles du fruit et du papillon suggèrent que la quantité nutritive est le principal déterminant de la taille des pyrales adultes. Toutefois, la variété Takermoust, aux fruits relativement courts mais charnus qui présentent des taux d'infestation élevés dans nos échantillons, pourrait aussi fournir des dattes plus efficaces pour la croissance des papillons, et la variété Bayd-Hmam, aux petits fruits, des dattes moins efficaces pour une telle croissance. Nous ne pouvons ainsi pas exclure que des différences de qualités nutritives participent à expliquer nos observations. Le fait que les dattes Takermoust soient parfois attaquées précocement pourrait, en outre, laisser plus de temps aux larves présentes dans de jeunes fruits pour se développer et atteindre de plus grandes tailles.

Nos observations répétées nous ont par ailleurs permis de constater que la teinte de la pyrale dépend de la couleur de la datte. La datte contient un mélange de pigments, notamment de nombreux caroténoïdes et flavonoïdes, qui détermine sa coloration. Il se pourrait alors que ce soit cette composition pigmentaire, qualitative et quantitative, qui détermine la variation de teinte des papillons. L'absorption des pigments par la chenille serait donc suivie d'un catabolisme très limité.

Le patrimoine phøenicicole algérien est confronté à de nombreux problèmes phytosanitaires. Le ver de la datte E. ceratoniae est l'un des déprédateurs les plus rencontrés, qui cause des dégâts considérables à la récolte tant du point de vue qualitatif que quantitatif. Il existe plusieurs types de lutte contre cette pyrale. Nous recommandons de favoriser les méthodes biologiques, les mieux adap- tées à un écosystème aussi fragile et aussi complexe que la palmeraie. Ainsi, pour la production de dattes, nous utilisons déjà les variétés de palmiers dattiers dont les fruits sont les moins infestés Mais nous avons aussi recours à certaines variétés plus et précocement infestées pour servir de bouclier aux arbres les plus productifs. Il a été constaté que cette technique, qui consiste à planter une variété très infestée telle que Takermoust en lisière des palmeraies constituées d'autres variétés, concentre les attaques sans augmenter la population totale de pyrales, et protège donc ces dernières variétés. Elle devra toutefois, dans les prochaines années, être associée à d'autres techniques pour augmenter l'efficacité de la lutte biologique. Des lâchers de trichogrammes, Hyménoptères parasitoïdes oophages, devront ainsi être entrepris dans les palmeraies les plus attaquées, cela dès le stade de grossissement des fruits s'il existe des variétés précocement infestées (Idder, 1984). Il faudra enfin protéger les autres parasitoïdes que nous avons recensés en évitant tout traitement insecticide à des moments sensibles. Ceux-ci comprennent les Braconidae Phanerotoma flavitestacea Fisher et $P$. planifrons Nees, qui sont des parasitoïdes ovolarvaires, ainsi que le Braconidae et l'Ichneumonidae Habrobracon bebetor Say et le Nemeritis canescens Gravenhorst, qui sont des parasitoïdes larvaires.

\section{Références}

Belguedj M. Les ressources génétiques du palmier dattier. Caractéristiques des cultivars de dattiers du Sud-Est du Sahara algérien. Dossiers-Documents-Débats $\mathrm{N}^{\circ} 1$. Alger : éditions INRAA, 2002.

Benaddoun A. Étude bio-écologique d'Ectomyelois ceratoniae (Lepidoptera-Pyralidae) à Ghardaïa. Mémoire ingénieur, INA EI Harrach, Alger, 1987.

Ben Khalifa A. Ressources génétiques du palmier dattier (Phoenix dactilyfera L.) et la lutte contre la fusariose. Organisation de la variabilité des cultivars du palmier des palmeraies du Sud-ouest algérien. Thèse de magister, ENS Kouba, Alger, 1989.

Bouguedoura N. Connaissance de la morphogenèse du palmier dattier (Phoenix dactylifera). Etude in situ et in vitro du développement morphogénétique des appareils végétatif et reproducteur. Thèse de doctorat d'État, USTHB, Alger, 1991.

Cossé AA, Endris JJ, Millar JG, Baker TC. Identification of volatile compounds from fungusinfected date fruit that stimulate upwind flight in female Ectomyelois ceratoniae. Entomol Exp Appl 1994 ; 72 : 233-8. 
Doumandji SE. Biologie et écologie de la pyrale des caroubes dans le Nord de l'Algérie, Ectomyelois ceratoniae Zeller (Lepidoptera-Pyralidae). Thèse doctorat ès science, université de Paris VI, 1981

Doumandji-Mitiche B. Contribution à l'étude bio-écologique des parasites et prédateurs de la pyrale des caroubes Ectomyelois ceratoniae en Algérie en vue d'une éventuelle lutte biologique contre ce ravageur. Thèse de doctorat ès science, université de Paris VI, 1983.

Giovanni G. Note sur les variétés de dattier cultivées en Algérie. Alger: éditions INRAA, 1969.

Haddad L. Quelques données sur la bio-écologie d'Ectomyelois ceratoniae dans les régions de Touggourt et Ouargla, en vue d'une éventuelle lutte contre ce déprédateur. Mémoire ingénieur, ITAS, Ouargla, 2000.
Hannachi S, Khitri D. Inventaire et identification des cultivars de dattiers dans la cuvette de Ouargla: organisation de la variabilité. Mémoire ingénieur agronome, INFSAS, Ouargla, 1991.

Hannachi S, Khitri D, Ben Khalifa A, Brac de la Périère AL. Inventaire variétal de la palmeraie algérienne. Rouiba (Algérie) : Ed. Anep, 1998.

Idder A. Inventaire des parasites d'Ectomyelois ceratoniae Zeller dans les palmeraies de Ouar gla et lâchers de Trichogramma embryophagum Hartig contre cette pyrale. Mémoire ingénieur agronome, INA EI Harrach, Alger, 1984

Idder MA. Aperçu bioécologique sur Parlatoria blanchardi Targ. (Homoptera, Diaspididae) en palmeraies de Ouargla et utilisation de son en nemi Pharoscymnus semiglobosus Karsh. (Coleoptera, Coccinellidae) dans le cadre d'un es sai de lutte biologique. Thèse de magister en sciences agronomiques, INA El-Harrach, Alger, 1992
Le Berre $M$. Mise au point sur le problème du ver de la datte Myelois ceratoniae Zeller. Bull Agr Sahar $1978 ; 1: 1-35$

Munier P. Le palmier dattier. Paris: Ed. Maisonneuve et Larose, 1973.

Pintureau B, Daumal J. Les Ectomyelois de I'ancien Monde (Lepidoptera-Pyralidae). Bull Soc Entomol Fr 1979; 84 : 84-8.

Raache A. Étude comparative des taux d'infestation de deux variétés de dattes (Deglet-Nour et Ghars) par la pyrale des dattes Ectomyelois ceratoniae (Lepidoptera-Pyralidae) dans deux biotopes différents (palmeraies moderne et traditionnelle) dans la région de Ouargla. Mémoire ingénieur, ITAS, Ouargla, 1990.

Wertheimer M. Un des principaux parasites du palmier dattier: le Myelois decolor. Fruit 1958; $13: 109-28$ 